Images du travail, travail des images

$9 \mid 2020$

L'apprentissage et l'enseignement professionnel en images

\title{
La technographie de Charles Dekeukeleire. Le rôle du cinéma dans la civilisation machinique
}

Charles Dekeukeleire's technography. The role of film in the construction of the industrial civilisation

Mathilde Lejeune

\section{OpenEdition}

\section{Journals}

Édition électronique

URL : http://journals.openedition.org/itti/496

DOI : $10.4000 /$ itti.496

Éditeur

Université de Poitiers

Référence électronique

Mathilde Lejeune, «La technographie de Charles Dekeukeleire. Le rôle du cinéma dans la civilisation machinique ", Images du travail, travail des images [En ligne], 9 | 2020, mis en ligne le 01 septembre 2020, consulté le 14 avril 2021. URL : http://journals.openedition.org/itti/496 ; DOI : https://doi.org/ $10.4000 /$ itti.496

Ce document a été généré automatiquement le 14 avril 2021

Images du travail, travail des images 


\title{
La technographie de Charles Dekeukeleire. Le rôle du cinéma dans la civilisation machinique
}

\author{
Charles Dekeukeleire's technography. The role of film in the construction of the \\ industrial civilisation
}

Mathilde Lejeune

\section{Introduction}

1 Le réalisateur belge Charles Dekeukeleire (1905-1971) a développé au long de la décennie 1940 la notion de "technographie", un terme qui interroge par son implication aussi brève qu'intense dans ses écrits théoriques. Élevée au rang de solution la plus appropriée aux problématiques engendrées par la modernité, la technographie se présente comme un vaste projet social utopique assigné au cinéma. $\mathrm{Si}$ elle désigne la production concrète de films industriels, elle semble cristalliser les enjeux d'une théorie plus large du cinéma comme moyen de construction, par la technique, des cadres spirituels de la civilisation machinique.

2 Aujourd'hui inconnu de la plupart, le cinéaste est pourtant considéré comme l'une des figures fondatrices de tout un art belge du film, justifiant d'une production aussi conséquente que diverse. Il s'oriente à la fin des années 1920 vers un cinéma expérimental qui fera véritablement sa renommée ${ }^{1}$ puis, exception faite de deux fictions $^{2}$, part dès les années 1930 dans la direction du documentaire de commande dont il tire environ quatre-vingts travaux jusqu'en 1962. Bien qu'ils représentent la grande majorité de son œuvre, ces films participent de son effacement dans l'histoire ${ }^{3}$. D'après les critères qu'il a mis en place, on y rencontre des films sur l'art, l'histoire, l'urbanisme, le tourisme et le folklore, puis l'industrie". Les films de commande présentent au fil des années un glissement depuis une esthétique encore en partie expérimentale, à une image de plus en plus institutionnalisée - résultat d'un amenuisement des libertés artistiques accordées par les commanditaires. Cependant, 
des épisodes poétiques traversent les œuvres, quelle que soit leur époque, formant un effet de résonnance esthétique et thématique à l'intérieur de sa filmographie. Les productions, de tous types, font systématiquement état d'un rapport à la machine particulier, dont on peut dire qu'il définit le style de Dekeukeleire. Dès lors, une vision chronologique de son œuvre pourrait apparaître comme problématique. Celle-ci cherche à construire un cadre spirituel autour du cinéma qui, depuis les années 1930 jusqu'aux années 1960, s'établit comme une seule et même pensée, sans cesse redéfinie à travers l'esthétique des films.

3 La production écrite du réalisateur se situe, elle aussi, à la croisée de nombreux champs, s'attachant tout autant à approfondir de manière obsessionnelle cette pensée des débuts. Ses idées, qu'il dévoile selon ce qu'il appelle une « méthode intuitive », sont intra-communiquantes. Elles apparaissent d'emblée éclatées, souvent inabouties et rendent délicat l'accès à ses réflexions, qui restent majoritairement abstraites. Elles se dévoilent à travers quelques articles, des notes personnelles réunies sous la forme de carnets $^{5}$ et deux ouvrages publiés ${ }^{6}$. Chaque pan de son discours tend vers ce que Dekeukeleire nomme la "pensée machinique», qui désigne autant le cinéma comme moyen d'écriture mécanique (par le montage), que l'archétype d'une pensée moderne issue du machinisme. Il s'efforce de faire communiquer ces deux dimensions, le premier devenant le moyen de construire le second en atteignant les foules par le canal des sens. Dans ses ouvrages, le terme de "technographie » désigne ce processus en s'appliquant aux films sur le travail industriel.

4 Présentée comme l'enjeu principal de la réflexion publiée de Dekeukeleire, la notion n'a paradoxalement bénéficié que d'un maigre rayonnement, à peine commentée par ses lecteurs. Elle doit probablement ce désintérêt à son hybridité, prise dans un réseau complexe de significations autant pratiques que théoriques qui la rendent indissociable de ce que l'on pourrait aisément définir comme l'œuvre spirituelle de toute une vie. La notion s'ancre en outre dans un contexte de pensée précis, se situant à la croisée de théories contemporaines de Dekeukeleire, tout particulièrement celles de Dziga Vertov (sur le plan social) et de Jean Epstein (sur le plan spirituel).

5 En vue de poser les jalons d'une définition de la technographie selon Dekeukeleire, cet article se propose d'analyser les divers contours de cette notion, dans un effort de contextualisation théorique et pratique. Depuis la vocation sociale du cinéma jusqu'à la pratique du film industriel, un portrait idéologique et méthodologique du parcours du réalisateur sera tracé. Un détour par le documentaire social s'imposera, pour finalement faire état du glissement de la notion de technographie vers le champ purement spirituel.

\section{Dekeukeleire et le machinisme : l'éducation par la technographie}

Dès les années 1930, le cinéaste explore la question sociale, qu'il développe au sein de deux ouvrages théoriques et d'une dizaine d'articles consacrés à son art de prédilection. En parallèle des écrits publiés, ces réflexions traversent avec obstination et presqu'invariablement ses carnets de notes personnels. Jusqu'au début des années 1960, il s'acharne à saisir les cadres de ce que l'on pourrait définir comme l'essence de son temps, l'ère des machines. Le médium cinématographique, pris dans son contexte 
social, devient un outil (technique) d'analyse du monde (sensible) doté d'un rôle actif et civilisateur.

7 La révolution industrielle, constate-t-il, a brutalement changé la vie occidentale. Le mode de production artisanal s'efface au profit d'une spécialisation toujours accrue des métiers; de laquelle surgit et croît l'individualisme, faisant obstacle à toute cohésion sociale. Devenus les rouages d'une mécanique fragmentée au rythme millimétré, les hommes et femmes du peuple subissent la modernité de plein fouet : ils perdent pied, ne saisissant plus tout à fait les liens qui se nouent entre leurs diverses tâches. Le travail à l'usine semble s'éloigner de toute finalité sociale ou spirituelle: «comme à Babel, nous parlons la même langue sans nous comprendre » (Dekeukeleire 1942 : 65). Il est urgent, selon le cinéaste, de reconstruire des idéaux communs.

Dekeukeleire pose en principe que toutes les civilisations se construisent selon les mêmes bases que sont "une économie, un art, des croyances, une pensée " (Dekeukeleire $1947: 9)$. Entre chacune de ces composantes se tissent des liens étroits qui reposent, eux, sur la technique : elle répond aux besoins d'une économie et permet à l'art de naître. Ce dernier alimente des croyances (par exemple religieuses, mais plus largement culturelles), lesquelles évoluent en postulats et forment la pensée d'une époque. La civilisation moderne, pour le réalisateur, en est au stade du tâtonnement. Il lui reste à construire ses propres croyances (ou rêves) par l'art que ses techniques engendrent.

9 En exprimant l'atmosphère de constant devenir qu'incarnent les révolutions technologiques et sociales, le cinéma devient, dans ses mots, "l'art-clef» du machinisme : « ce qui nous attire, c'est de voir ce qui normalement échappe à nos yeux. Le regard du cinéma dépasse notre horizon habituel » (Dekeukeleire $1947: 15)$. Douée d'une vue et d'une ouïe décuplées, la représentation mécanique offre à son spectateur l'expérience d'un point de vue inédit et objectivé, dépassant les perspectives humaines - du plan microscopique au plan le plus rapproché. Le regard optique, extériorisé, adopte alors quelque propriété analytique que le montage des images entre elles vient renforcer: non seulement déconstruit-on les espaces, mais l'on peut également décortiquer le temps, le raccourcir ou l'étirer.

10 Puisqu'elle déplace le regard à travers l'expérience incarnée des images, l'expression technique du film lui permet d'édifier les fondements d'un nouveau mode de perception qui naît avec l'industrialisation : il devient l'outil idéal pour amener le peuple à une unité spirituelle qui s'est a priori perdue dans l'intervalle entre travail artisanal et industriel. Pour prendre pleinement conscience de ses propres structures et atteindre un stade d'harmonie généralisée, la société doit selon Dekeukeleire faire du travail industriel son rêve collectif :

« Si chacun se donne entièrement à son travail et en fait le centre de sa vie spirituelle, notre civilisation croîtra rapidement vers sa maturité. Une technique propre à faciliter ce don du travail naît spontanément dans chaque civilisation. Le cinéma, sous la forme que nous avons appelée technographie, nous apporte cette technique » (ibidem : 110).

11 Comme évoqué en introduction, la technographie désigne dans un premier temps le documentaire industriel. Elle représente la partie la plus conséquente de la pratique filmique de Dekeukeleire et, exclusivement commanditée par les industries qu'elle promeut, entend décrire et valoriser le travail des ouvriers à l'usine en documentant leurs gestes techniques. Le terme, qui disparaît des notes du réalisateur à peine sept 
ans après son apparition en 1942 (fig. 1), s'avère paradoxalement central dans ses théories sur le cinéma: les réflexions de ses ouvrages publiés convergent à l'unisson vers la technographie, que le discours tend à confondre avec le documentaire et, bien souvent, le cinéma.

Figure $1:$ Indexation de la « technographie »

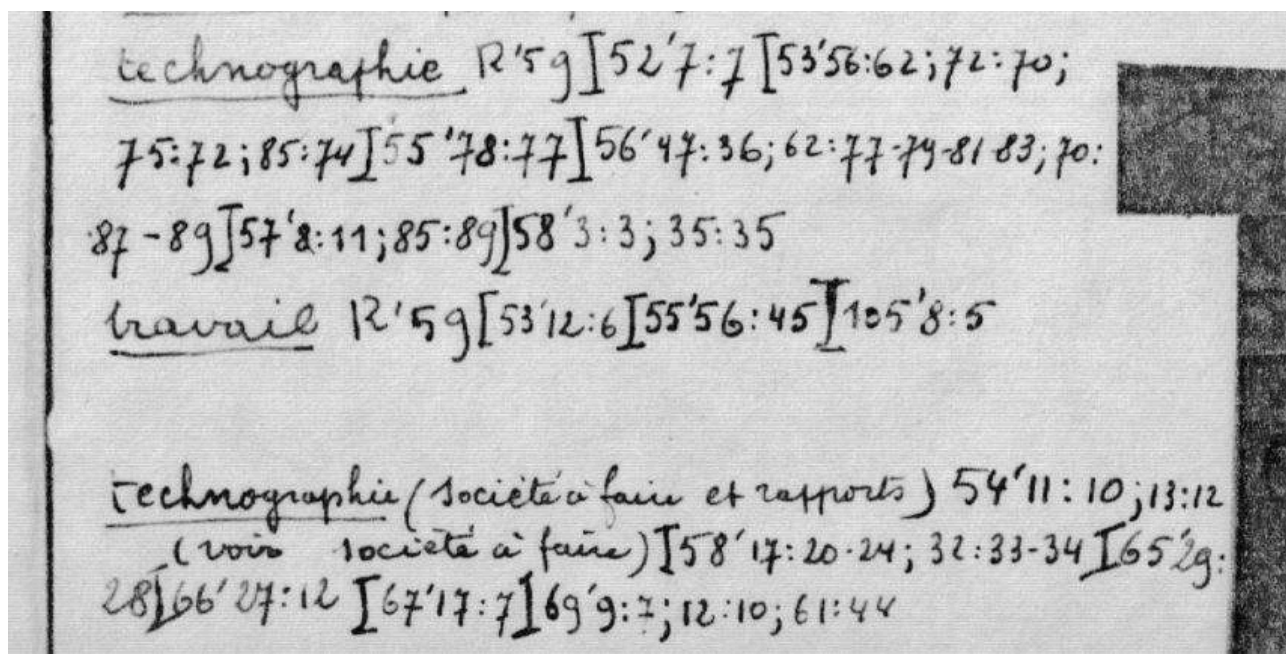

Ces références renvoient aux carnets de notes $n^{\circ} 52$ à n69, remplis entre 1942 et 1949 par Dekeukeleire (Index R des carnets de Charles Dekeukeleire, 1962, non paginé)

(c) Cinémathèque royale de Belgique. connaît ni industrie, ni fonds publics propres avant les années 1950. Les années 1920 voient s'ériger la Société Nationale de Propagande et de Cinématographie, laquelle a pour « objectif de faire connaître l'activité de la Belgique [...] et cela " sous forme d'un magazine cinégraphique commercial et industriel » (Aubenas 1999: 19). Dans le prolongement de cette structure, quelques institutions privées et publiques commandent ponctuellement la production de films institutionnels à partir des années 1930 à certains cinéastes de renom dont Henri Storck ${ }^{7}$ et Charles Dekeukeleire, qui travaillaient jusqu'alors de manière indépendante. Ces films de commande "ont presque toujours un caractère pédagogique » (Sojcher 1999: 31), leur but étant avant tout de faire connaître le patrimoine et les industries belges au public à travers une expression artistique marquée, pour laquelle on aura laissé plus ou moins de liberté esthétique ${ }^{8}$.

films produits par Dekeukeleire se situent essentiellement dans ce cadre, et il semblerait que le cinéaste reprenne les impératifs commerciaux des commanditaires à l'avantage de ses propres idéaux. Il fantasme un film certes promotionnel, mais qui, par ses capacités à attirer les foules et à déformer la perception de la réalité, se donnerait pour mission de changer en profondeur le rapport de l'homme au monde en modifiant 
le regard que celui-ci porte sur le travail. Les films de commande deviennent alors un moyen pour mettre en œuvre l'idée d'un cinéma éducatif qui, dans les écrits, transparaît de manière peu concrète.

Comme une partie de ses contemporains, le projet d'une industrie et d'une économie pleinement destinées au cinéma belge préoccupe Dekeukeleire au sein de certaines conférences ${ }^{9}$, dont les réflexions se prolongent dans ses carnets. Il parvient à ouvrir des studios à Waterloo au cours des années 1950 (fig. 2).

Figure 2 : Schémas de construction pour les Studios des « Films de Belgique »

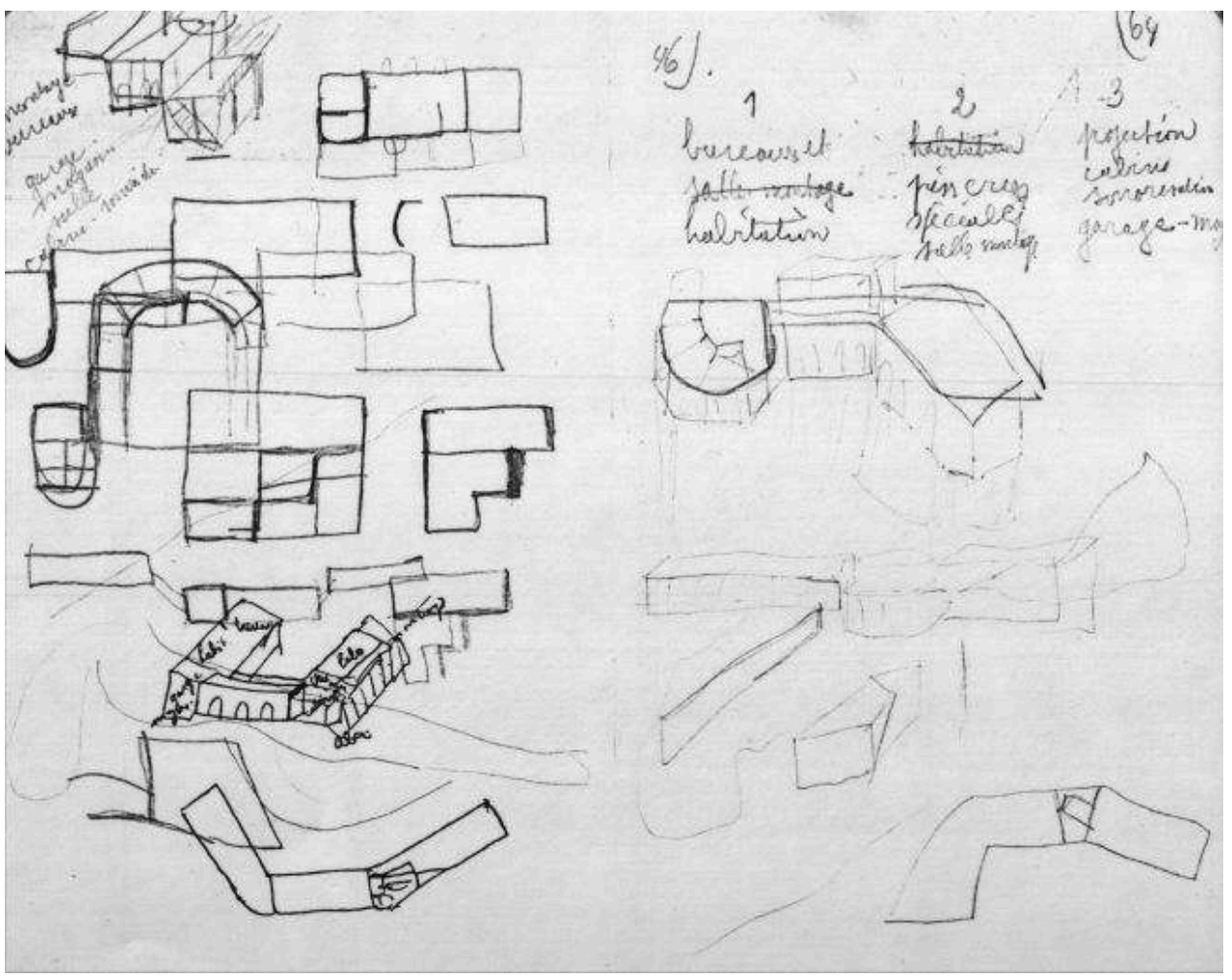

Ces plans sont les seuls qui existent dans les notes du réalisateur et qui permettent de comprendre la configuration de ses studios. Il envisage de trouver un château pour y installer son industrie, qu'il finit par acheter à Waterloo en 1954 (Carnet n69 de Charles Dekeukeleire, 5 juin 1949 - 21 novembre 1949, p. 64).

(c) Cinémathèque royale de Belgique.

L'opération, qui échoue au bout d'une petite année, n'est autre que l'application concrète de son idée de technographie. Ses notes personnelles font apparaître dans les années 1940 de véritables plans d'action pour la mise en place d'une société de production entièrement dédiée aux films technographiques. Tels qu'il les envisage, ceux-ci présentent un large spectre d'objets, tous concernant différents pans du travail industriel en Belgique. De la budgétisation qu'il redétaille sans cesse au parcours des différents thèmes dont doivent traiter les œuvres (fig. 3 \& 4) se dessinent les contours d'un processus tangible (autant qu'utopique) d'harmonisation sociale par le film industriel :

«Les travailleurs reçoivent, grâce à la technographie, la révélation de leurs gestes accomplis dans la monotonie [...]. Producteurs, ouvriers, consommateurs se rejoignent dans la contemplation des mêmes réalités modernes, des mêmes Images, du même rêve » (Dekeukeleire 1947 : 55). 
Figures 3 et 4 : Réflexions autour de la structure d'un « scénario technographique type »

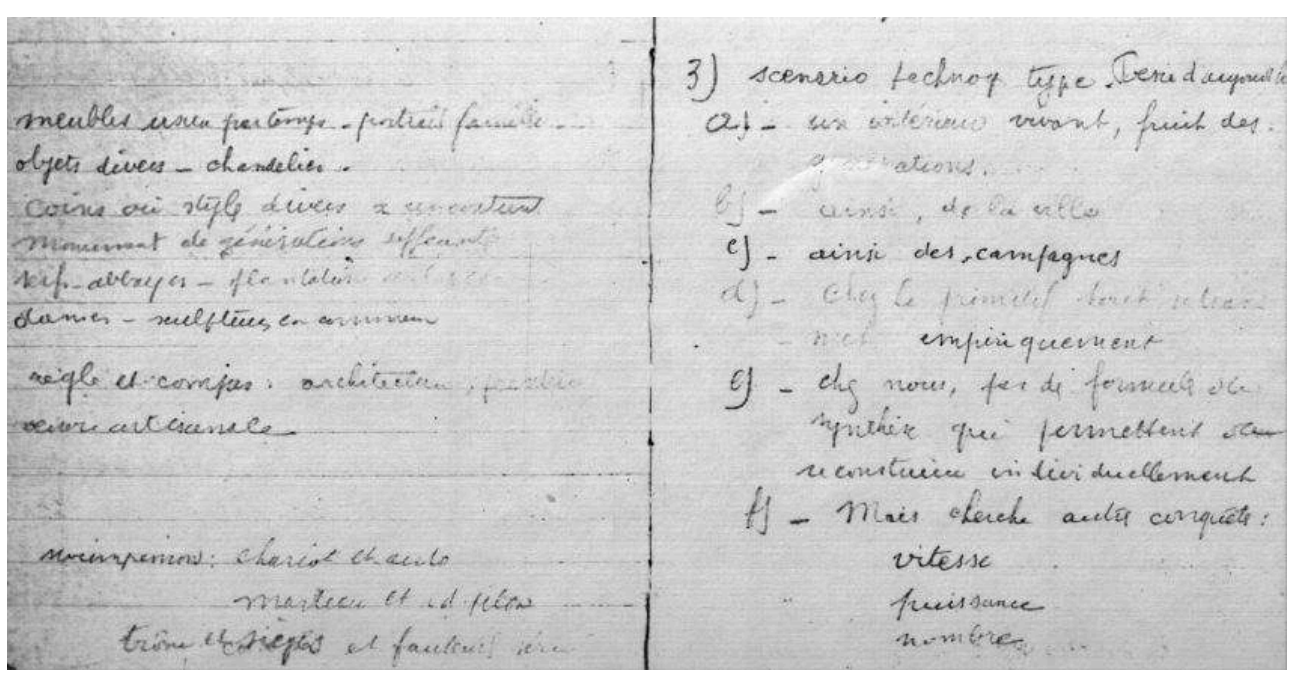

(c) Cinémathèque royale de Belgique.

\section{Figure 4}

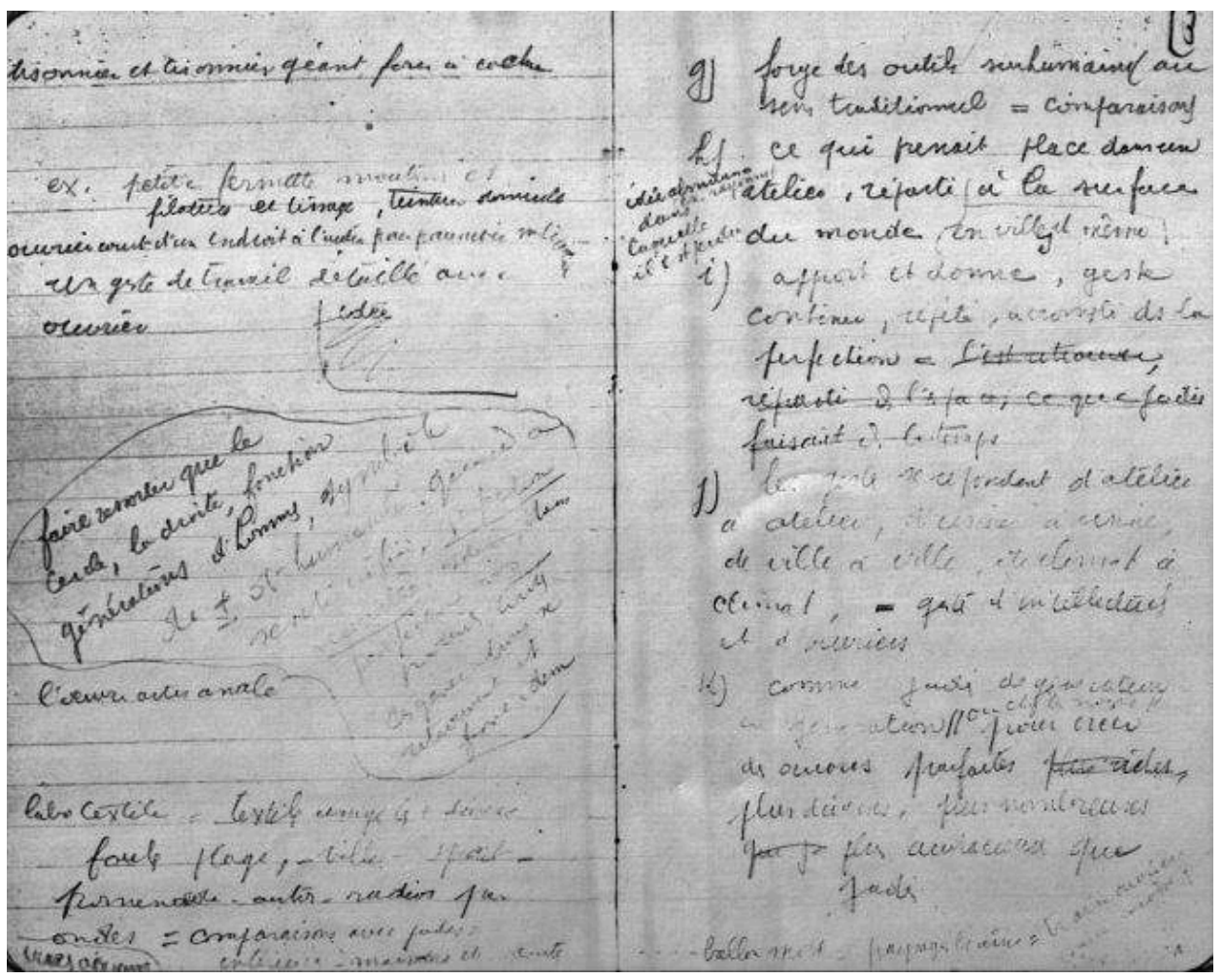

L'éclatement des divers éléments fait transparaître l'ambivalence des enjeux au départ du projet de Dekeukeleire qui, cherchant à saisir « tous » les aspects du travail industriel, entend porter un discours unifiant et totalisant sur les cadres d'une humanité moderne (Carnet $n^{\circ} 62$ de Charles Dekeukeleire, 4 août 1946 - 15 février 1947, p. 8-9).

(c) Cinémathèque royale de Belgique. 


\section{La caméra, vision objectivée d'une réalité sociale}

17 En insistant sur les dimensions analytique et éducative du film industriel, le discours du réalisateur pose au cinéma des questions de méthode de prise de vue et de montage qui, s'il les associe à la technographie des années 1940, concernent en premier lieu ses premières images documentaires - tournées dès les années 1930. Guidée par la vision optique, l'écriture cinématographique dépasse le cinéaste :

"J'ai appris ainsi qu'il faut se soumettre. Se laisser enrichir par l'appareil. Pour monter un film, il faut lentement, patiemment, se soumettre au document, apprendre ainsi tout ce que le sujet contient d'humanité indépendamment de l'impression qu'il vous fit à son premier contact. Le montage d'un film documentaire est [...] une lente maturation individuelle d'une sensibilité collective » (Dekeukeleire 1942 : 112-113).

La caméra aide le cinéaste à s'écarter de ses propres préjugés, à sortir de lui-même pour saisir véritablement l'essence de la réalité «brute » et contingente qui s'offre à la lentille focale. En dehors de toute construction préalable, la prise de vue est une opération d'observation désincarnée de laquelle résulte la vraie création - celle d'un montage intuitif : l'authenticité des images finales viendra de la capacité du cinéaste à rendre sensible la rencontre entre une réalité donnée et le regard de la caméra. obéissant alors au pouvoir de l'œil machinique, le réalisateur se pose a priori en traducteur quasi-objectif de la technique, révélant une dimension plus méthodologique de la notion de Dekeukeleire.

Ces postulats, s'ils ne transparaissent que très succinctement dans le discours du cinéaste, sont en lien direct avec une certaine veine du documentaire social: le vingtième siècle voit émerger avec l'enregistrement mécanique une véritable nécessité de documenter le quotidien. Intelligible de tous, le langage du cinéma incarne celui de la mémoire du monde, il possède le pouvoir de conserver la diversité des choses dans leur aspect éphémère. Parallèlement à une tradition scientifique qui l'accompagne dès sa naissance, le regard cinématographique s'envisage par ailleurs dans son caractère d'objectivité. La caméra devient un «sur-œil» (Aumont 2002: 62) capable de transcender les perceptions humaines, donnant lieu à diverses théorisations.

Parmi celles-ci se démarque celle de Dziga Vertov, documentariste et opérateur d'actualités filmées soviétique. Il présente une pensée du cinéma engagée (dans le communisme) et exprimée dans une méthode stricte qui, depuis les années 1920 jusqu'à nos jours, n'a cessé d'inspirer tout un cinéma militant. Théorisant sa propre pratique, Vertov introduit le radical "ciné-œil», traduction de kinoglaz: par son aspect mécanique, la caméra est pour lui un œil à l'objectivité impérieuse face auquel le cinéaste doit faire preuve d'un effacement total. Il doit s'efforcer, dans le montage, d'en restituer la vision sensible - en vue d'amener à la conscience du spectateur les aspects cachés de sa propre vie. Tout élément de mise en scène est banni de façon à capturer "sur le vif » diverses situations de la réalité quotidienne. Le matériel occupe à ces fins une place prépondérante : il doit être suffisamment maniable pour pouvoir à la fois se dissimuler au regard de ceux que l'on filme et se frayer un chemin dans les situations les plus physiquement inaccessibles.

21 Dekeukeleire s'inspire de Vertov, prenant connaissance de son travail au tout début des années 1930. À travers ses discours publics, le réalisateur énonce un engagement marqué : il se positionne contre toute pratique du studio ou du scénario, rejetant par-là 
l'idée d'une mise en scène. Ces postulats méthodologiques, cependant, ne se trouvent que très peu mis en œuvre dans sa pratique filmique qui, si elle exprime à ses débuts un radicalisme esthétique fort ${ }^{10}$, interroge quant à sa vocation sociale. Le film le plus politiquement engagé de Dekeukeleire est sans doute Witte Vlam (1930) ${ }^{11}$ - qui signifie Flamme Blanche. Par une méthode proche du "ciné-œil», le réalisateur filme une manifestation du parti populaire flamand depuis des points de vue internes (par un procédé de caméra participante) autant que très externes (depuis, notamment, le haut d'une tour). À ces images contingentes se confrontent brutalement des vues de la campagne et des gros plans d'ouvriers, dans une volonté de créer un dialogue sur les conditions du travail moderne. Bien que n'affichant pas de jeu d'acteur, il est peu probable - au vu de l'esthétique soignée des plans - que les individus aient été surpris par la caméra de Dekeukeleire qui, par-là, interroge l'impérieuse objectivité du regard mécanique. En outre, le récit fictionnel d'un boucher poursuivi par des policiers se profile dans les contours de l'œuvre, ajoutant une valeur symbolique aux images documentaires autant qu'il détruit l'idée d'une «non mise-en-scène " à laquelle Dekeukeleire semblait adhérer en premier lieu.

Si le documentaire a pour but principal, chez le cinéaste, de dévoiler une vérité sociale objectivée par l'œil de la caméra, la technique de prise de vue est néanmoins effectuée par l'individu, c'est-à-dire socialement normée et dénuée d'une réelle neutralité. S'interrogeant sur la subjectivité de la syntaxe cinématographique, il nuance son propos: au ciné-œil, il répond par le "ciné-esprit» (Dekeukeleire 1932: 226), défendant l'idée d'une construction préalable au film à condition que celle-ci serve à apporter un regard critique aux documents qu'elle véhicule. En revenant de la sorte sur ses engagements techniques, Dekeukeleire ancre son discours dans une veine du documentaire social qui, si elle s'approche des idéaux de Vertov (comme le fera, également, un cinéma ethnographique), vise néanmoins à ré-affirmer la place de l'opérateur. En Europe, des réalisateurs tels - entre autres - qu'Henri Storck, Joris Ivens ou Jean Vigo (que Dekeukeleire côtoie) développent un regard volontairement subjectif :

« [Le] documentaire exige que l'on prenne position [...]. L'appareil de prise de vues sera braqué sur ce qui doit être considéré comme un document, et qui sera interprété, au montage, en tant que document. Bien entendu, le jeu conscient ne peut être toléré. Le personnage aura été surpris par l'appareil, sinon l'on doit renoncer à la valeur " document» d'un tel cinéma. Et le but sera atteint si l'on parvient à révéler la raison cachée d'un geste, à extraire d'une personne banale et de hasard sa beauté intérieure ou sa caricature, si l'on parvient à révéler l'esprit d'une collectivité d'après une de ses manifestations purement physiques » (Vigo $20: 226$ ).

Dans le prolongement de Witte Vlam, Dekeukeleire tourne en 1932 Visions de Lourdes ${ }^{12}$, un documentaire sur le célèbre pèlerinage commandité par les JOC (Jeunesses Ouvrières Chrétiennes) de Belgique - lesquelles censureront l'œuvre jugée trop critique vis-à-vis de la religion catholique. Il y reprend les convictions de son " ciné-esprit ", similaire au documentaire social décrit par Vigo. Muni d'une caméra qu'il intègre au cœur des événements sans la cacher, il ne nourrit son film que d'images documentaires prises sur le vif, montrant deux réalités de l'événement : au rituel des pratiquants répond celui du mercantilisme. 
Ce média ne peut être affiché ici. Veuillez vous reporter à l'édition en ligne http:// journals.openedition.org/itti/496

La caméra, adoptant des points de vue a-humains, se veut objectivante. Le regard du spectateur se trouve néanmoins orienté par un montage qui, lui, mobilise les plans dans un discours critique sur les rapports entre religion et commerce à Lourdes (fig. 5).

Ciné-œil, documentaire social et ciné-esprit prolongent à l'unisson les enjeux analytiques et didactiques véhiculés par l'œil objectivant de la caméra. Captant ce qui est invisible à l'œil nu, la technique du cinéma - orientée par la sensibilité du cinéaste a le pouvoir de révéler les dynamiques internes de la réalité sociale. Elle répond alors à l'idéologie d'un film qui éduque le peuple et que l'on trouve au départ du projet technographique de Dekeukeleire.

\section{Une méthodologie du film technographique ?}

27 Face à un discours qui porte le documentaire et la technographie sur un même plan, les notes personnelles de Dekeukeleire interrogent la mise en pratique d'une telle ligne de conduite dans le champ du film industriel. À l'absence de scénarios répondent, en amont des tournages, des épisodes de préparation plus ou moins longs où s'alternent des recherches contextuelles historiques, économiques ou simplement pratiques (fig. 6) avec la planification de vues à filmer et de découpages à effectuer (fig. 7).

Figures 6 et 7 : Préparations préliminaires pour Métiers d'Art de Flandre et de Wallonie, Charles Dekeukeleire, 1950

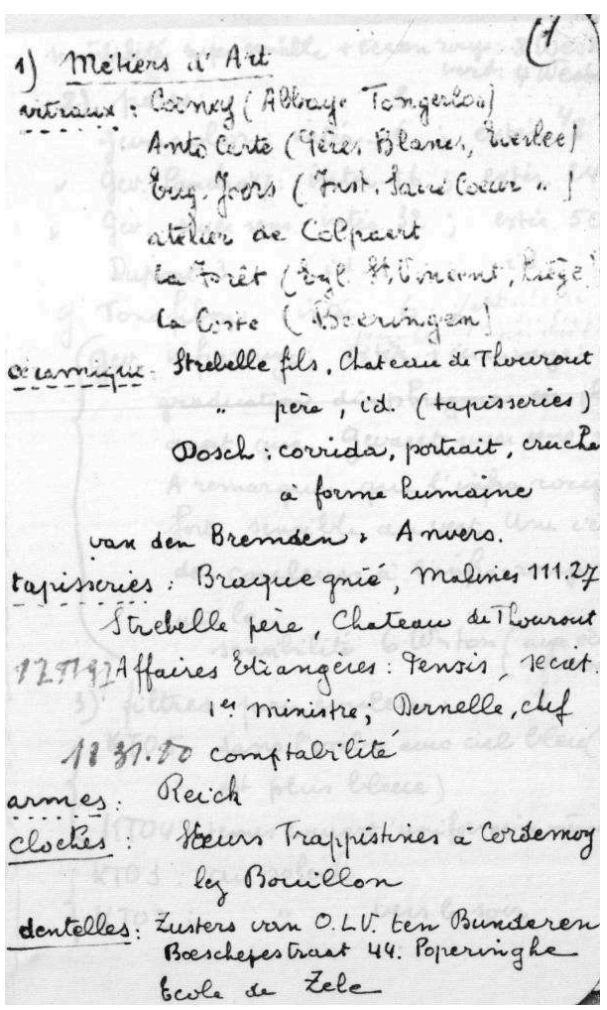

(C) Cinémathèque royale de Belgique 


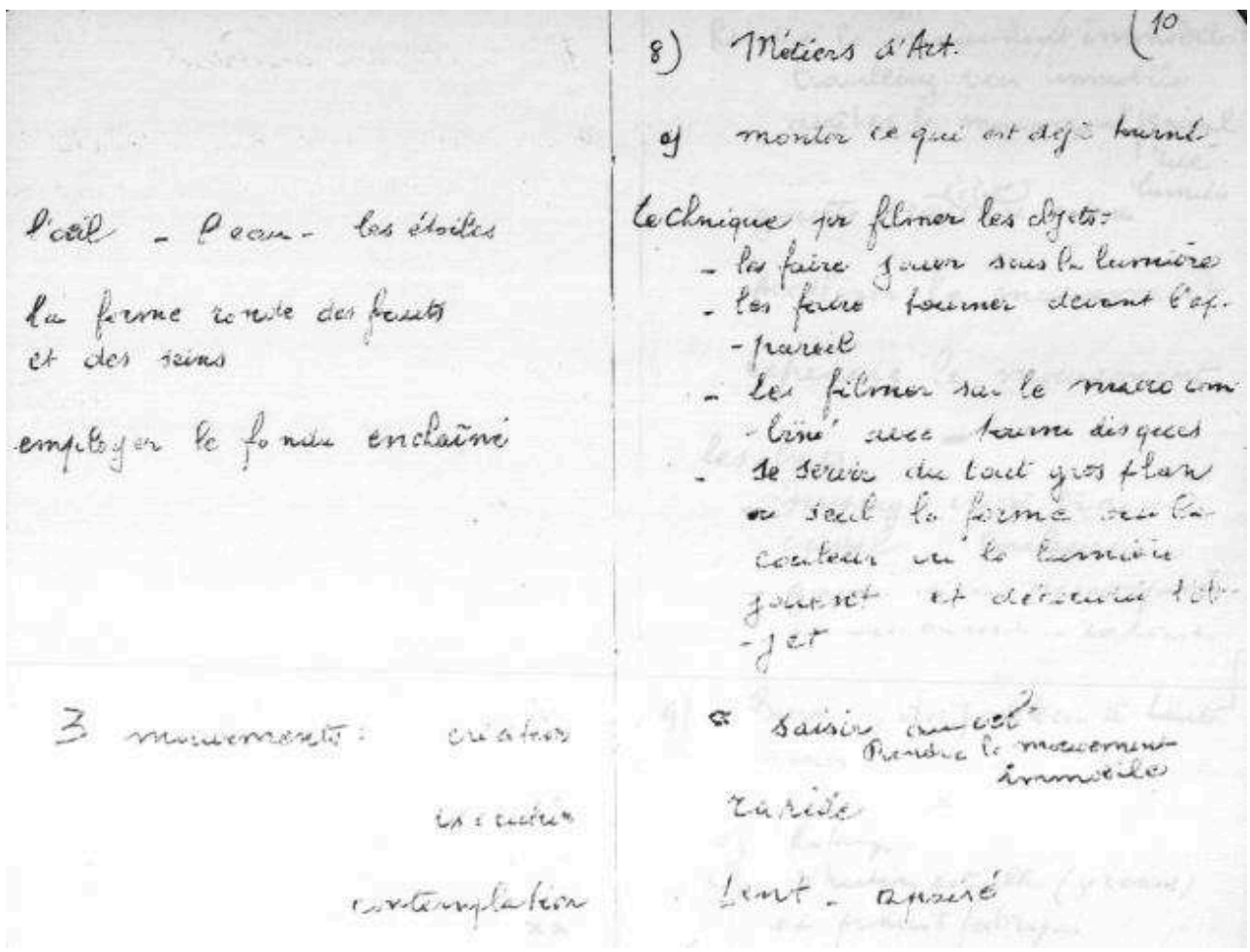

Le réalisateur prévoit dans un premier temps les artisans qu'il va contacter pour son film, puis réfléchit aux différentes techniques qu'il utilisera pour retranscrire son propos, qu'il veut poétique. On remarque d'emblée la mise en scène inhérente aux futures images, qui mettront l'accent sur la valorisation des objets filmés plus que ne le ferait une prise de vues «sur le vif » (Carnet n 67 de Charles Dekeukeleire, 1er novembre 1948 à février 1949, p. 1 et p. 10).

(c) Cinémathèque royale de Belgique

Ce système s'applique à la quasi-entièreté de la production de Dekeukeleire toutes catégories confondues - la régularité et l'assiduité de la préparation des films étant très aléatoires et dépendant probablement du temps dont disposait le cinéaste. Au moment du tournage, celui-ci annotait les différentes prises de vues (fig. 8) pour ensuite les remanier plusieurs fois dans ses carnets afin d'arriver à un montage final (fig. 9). 
Figure 8 : Annotations au moment du tournage de Métiers d'Art de Flandre et de Wallonie

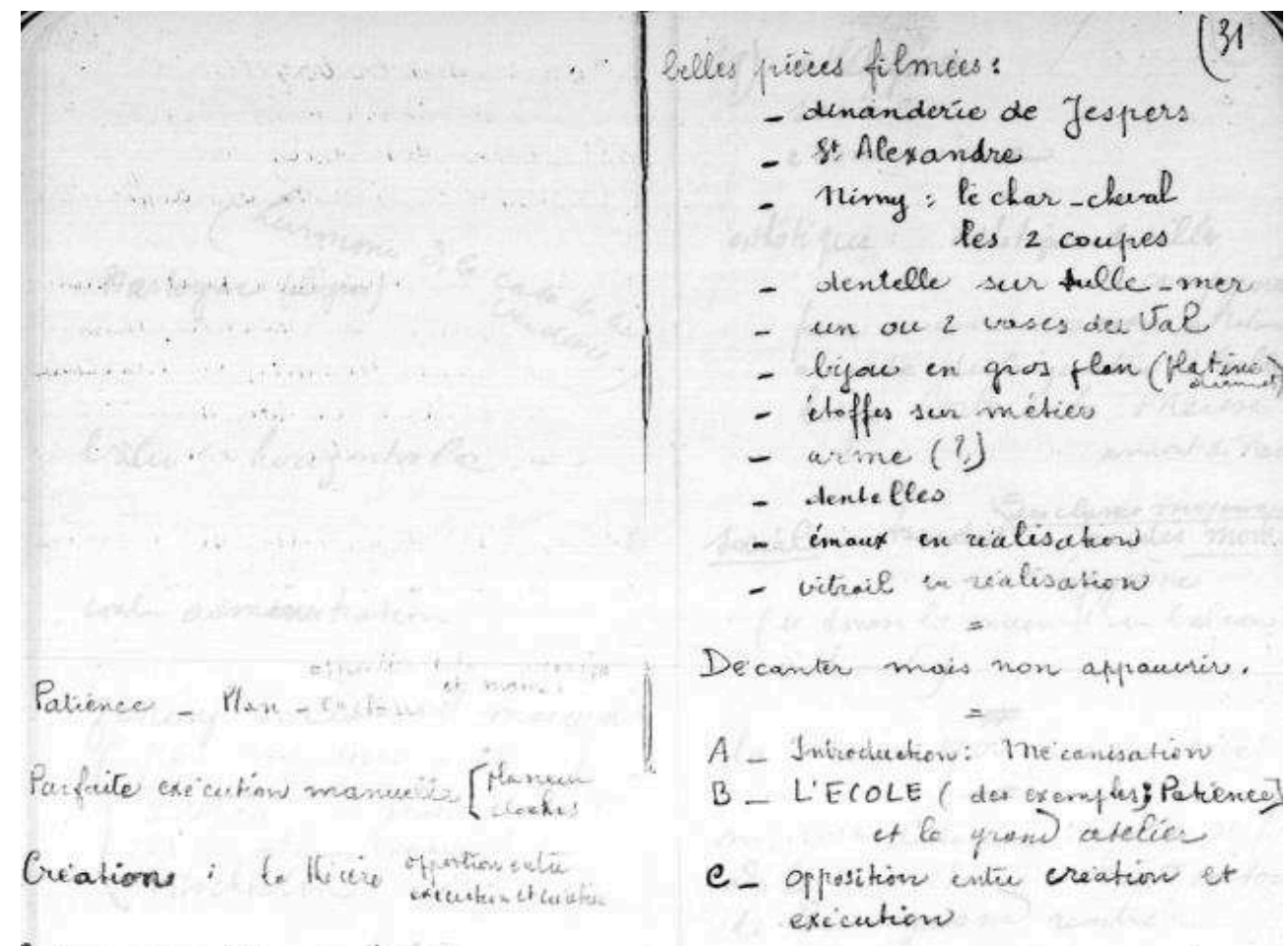

Jmagination - Mowito theed.

Les prises de vues que Dekeukeleire choisit l'amènent à réfléchir à la structure de son film et à l'atmosphère qui y sera véhiculée (Carnet $n^{\circ} 67$, p. 31)

(c) Cinémathèque royale de Belgique 
Figure 9 : Évolution du montage de Métiers d'Art de Flandre et de Wallonie

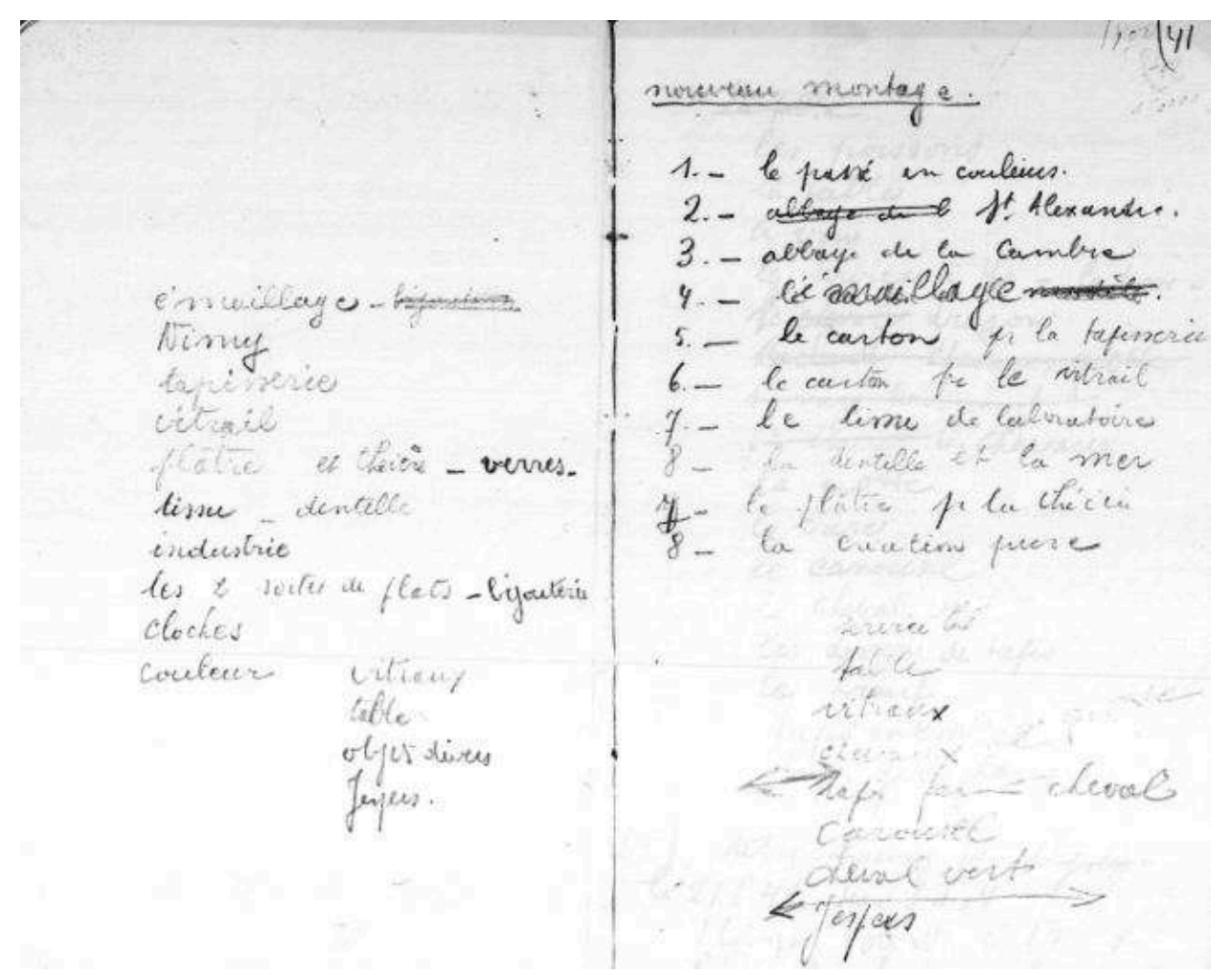

Le montage envisagé par Dekeukeleire évolue au gré du tournage, montrant une influence certaine de la prise de vue sur la structure finale, en accord avec la méthodologie que développe Dekeukeleire (Carnet $n^{\circ} 67$, p. 14 et p. 41).

(C) Cinémathèque royale de Belgique

29 L'analyse des notes techniques, si elle permet de voir des changements dans l'évolution des travaux, met néanmoins en branle l'idéologie de Dekeukeleire. Celle-ci s'appliquant chez lui au film (industriel) de commande, elle répond non plus aux exigences impérieuses de la machine mais à celles de l'État ou des entreprises, ce que la préparation préalable aux tournages appuie. Les traces de collaborations entre le cinéaste et les commanditaires ayant aujourd'hui en grande partie disparu, il est difficile d'évaluer leurs méthodes de travail, le rôle des entreprises dans la production des films et le degré de liberté artistique alloué à Dekeukeleire. Filtrée par les besoins promotionnels des uns et des autres, la réalité soi-disant brute du peuple au travail acquiert, dans l'esthétique finale des filmstechnographiques, des aspects propagandistes.

Ceux-ci se structurent généralement de la même manière: les techniques qu'ils entendent décrire sont géographiquement et historiquement contextualisées, dans le but d'une valorisation des différentes industries belges. On voit ensuite s'alterner divers appareils en action (fig. 10) avec des humains travaillant à l'aide de ceux-ci (fig. 11). 
Figure 10 : Vue d'un métier à tisser en pleine action dans La laine, Charles Dekeukeleire, 1936

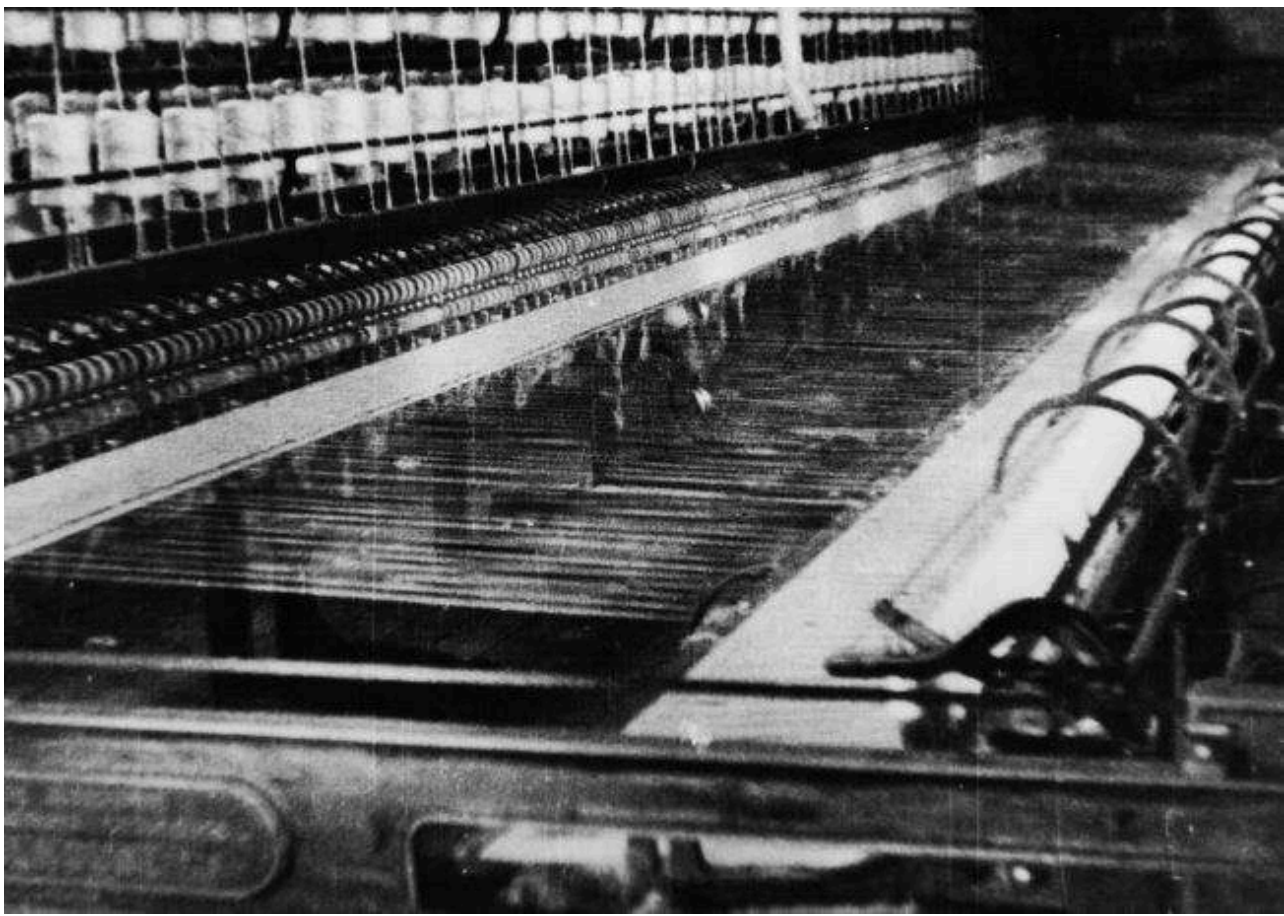

(C) Cinémathèque royale de Belgique

Figure 11 : Une ouvrière de l'industrie pharmaceutique dans Images du travail, Charles Dekeukeleire, 1938

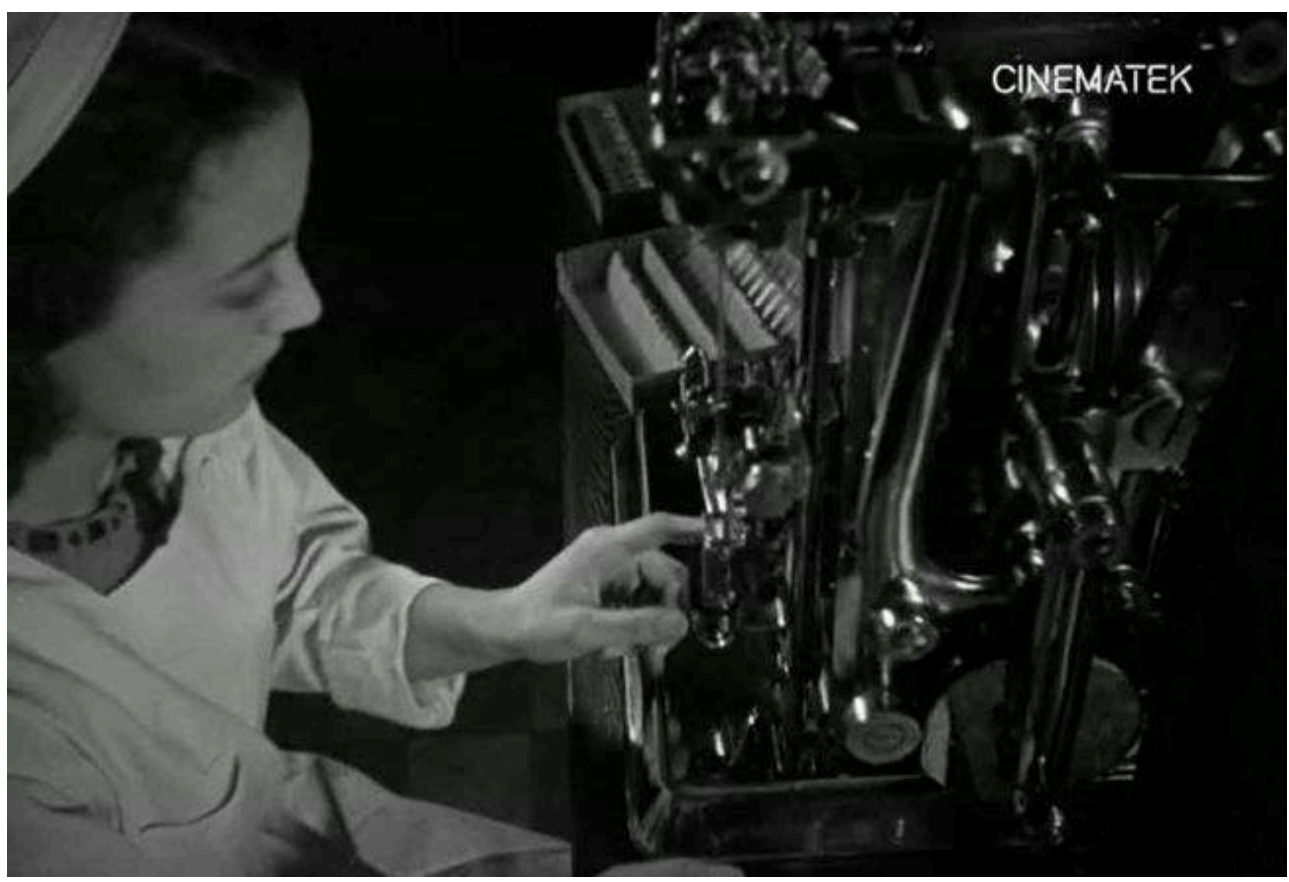

Capture d'écran effectuée à partir de la plateforme European Film Gateway.

(c) Cinémathèque royale de Belgique

Ces vues accompagnent chronologiquement les étapes de la production technique dans une volonté descriptive mais peu explicative. Les films technographiques se destinent à valoriser le fonctionnement général de l'usine, plus qu'à en comprendre les aspects 
techniques et sociaux. Différentes échelles de plan s'enchaînent le plus souvent par surimpressions dans un montage alterné qui permet à Dekeukeleire de rapprocher les mouvements des machines, que l'on voit s'accumuler poétiquement, et ceux des humains, dont on peut percevoir le rôle dans le déroulement de la production.

Figure 12 : Gros plan sur les bases du métier à tisser dans La laine

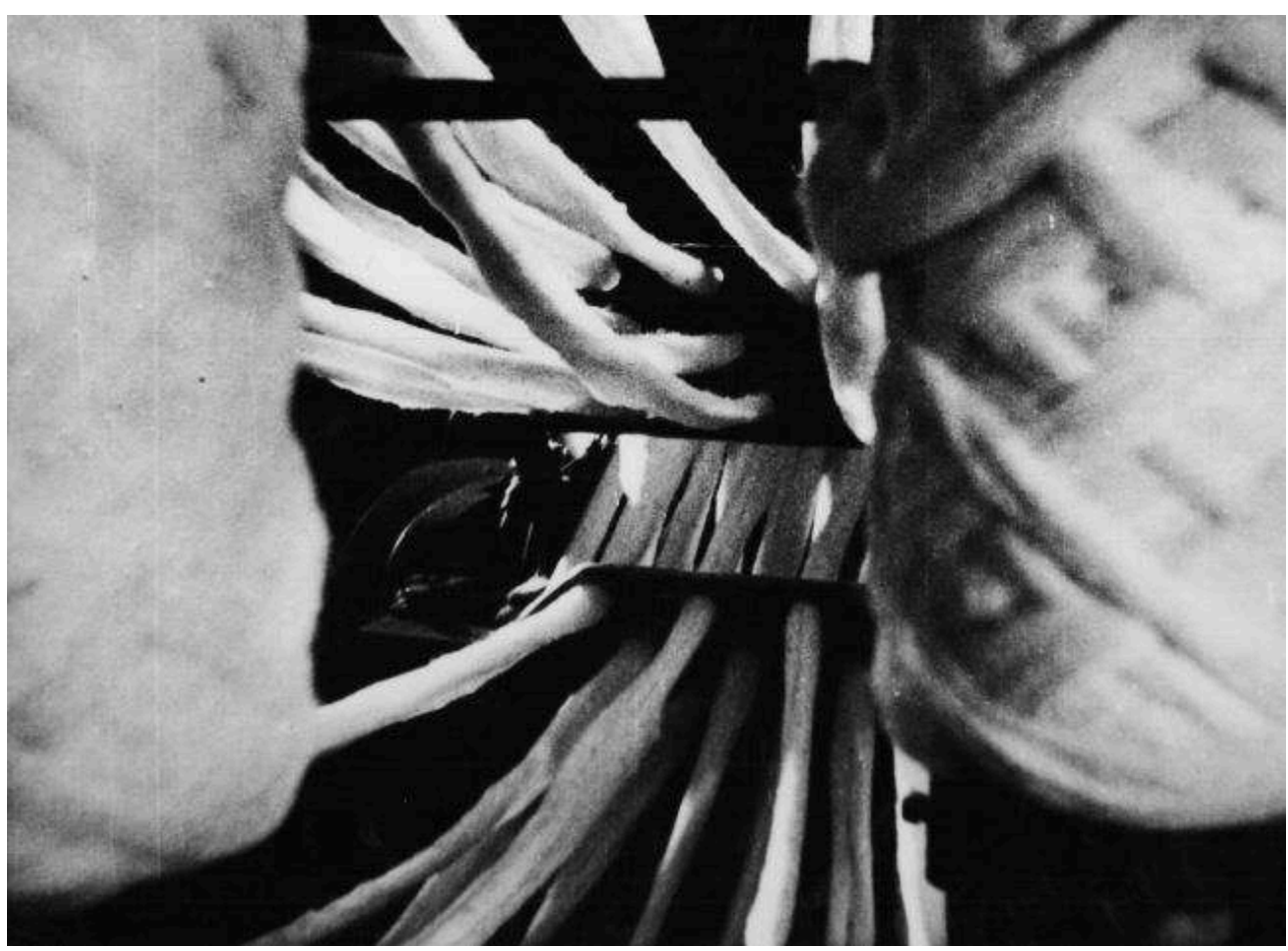

L'échelle de plan permet d'accéder à une partie difficilement accessible de la machine pour mieux en comprendre les rouages.

(c) Cinémathèque royale de Belgique

Le gros plan est très régulièrement mobilisé (fig. 12) pour montrer les structures métalliques en action. L'absence de distance donne une impression d'immersion à l'intérieur de leur mécanisme et tend à donner aux machines quelque caractère dramaturgique : elles sont au centre des films, magnifiées par le travail de lumière, elles en millimètrent le temps et l'espace, c'est autour d'elles que se centre l'attention. Dans une volonté prioritairement institutionnelle, l'image finale s'agrémente le plus souvent d'un commentaire vantant les bienfaits du travail industriel, du rôle que l'ouvrier y joue et du confort dont celui-ci bénéficie désormais (fig. 13). Mais l'on ne montre pourtant jamais l'ouvrier que par fragments, sa parole est tue au profit d'une machine mise en avant par la rythmique des gros plans. Ce regard, loin d'interroger la position sociale ou les difficultés quotidiennes du travailleur, lui reste extérieur et le réifie plutôt: sa main devient un outil pour prolonger la machine, l'ouvrier se transforme en rouage dans l'élaboration du produit final.

Ce média ne peut être affiché ici. Veuillez vous reporter à l'édition en ligne http:// journals.openedition.org/itti/496

Dekeukeleire ancre alors son discours dans une idéologie du progrès et un positivisme caractéristiques de son époque à l'égard des techniques industrielles, situant 
finalement la technographie, dans son usage pratique, en dehors du documentaire social duquel elle semblait se rapprocher dans les écrits. S'éloignant des méthodes engagées dans ses toutes premières œuvres documentaires, les films industriels intègrent une logique institutionnelle et commerciale propre aux productions de commande :

«Les films d'entreprise n'ont pas pour objectif de montrer les réalités concrètes du travail. Ils ont des objectifs commerciaux : ils mettent en scène de préférence les moyens de production ou l'outillage utilisés, au détriment du savoir-faire des ouvriers de l'entreprise. L'usine n'y est jamais montrée comme le lieu d'affrontement entre différentes catégories sociales aux intérêts divergents. À l'inverse est mise en valeur l'idée de culture d'entreprise. L'ouvrier est occulté par une mise en scène qui privilégie la fabrication de l'objet dont on vantera les mérites » (Rémillet 2009 : 28-29).

Ce glissement vers des films publicitaires ou institutionnels caractérise une majorité des documentaristes belges des années 1930. C'est en revanche dans le discours théorique de Dekeukeleire que sa singularité transparaît: loin de catégoriser les films technographiques comme purement institutionnels, il porte sur eux le même discours que sur le cinéma en général, leur assignant les mêmes missions sociales, tout aussi éducatives et analytiques.

Un paradoxe se crée dans l'intervalle entre postulats et empirisme: à défaut d'une pratique, la technographie telle qu'il la décrit tend largement vers la théorie, voire (encore) l'utopie. Cette hypothèse se confirme alors qu'il inscrit dans un carnet en 1944 qu'il ne s'agit pas pour lui « de découvrir une esthétique de la techno[graphie] - mais les principes qui doivent permettre à la techno[graphie] de découvrir promptement une "âme " d'aujourd'hui » (Dekeukeleire, 1943-1944: 65). La vocation sociale du cinéma se retrouve alors au sein des films sur un plan plus allusif que concret :

«Le geste préoccupe l'œil de Dekeukeleire. [...] Le projet du cinéaste est plutôt d'établir des équivalences métaphoriques, des analogies visuelles entre l'humain et la machine de façon à ce que chaque travailleur puisse voir son labeur quotidien [...] élevé à une dimension supérieure, spirituelle, dans laquelle l'harmonie et la symbiose prévalent. Rendre foi dans le travail en présentant les icônes de celui-ci. » (Lesuisse 1998 : 64)

Ne cherchant pas à décrire les gestes techniques ou à montrer la réalité sociale des usines, le discours que Dekeukeleire adopte à travers son montage tend vers l'essentialisme :

«La technographie [...] ne peut transcrire intégralement notre geste, elle doit le synthétiser, pour que nous puissions en prendre connaissance, le monter comme on dit en langage cinématographique. Elle doit rythmer l'ubiquité nécessaire des regards, elle doit découvrir le rythme selon lequel notre âme peut faire sienne cette ubiquité » (Dekeukeleire 1942 : 106).

Le geste de l'ouvrier devient allégoriquement celui d'une société (fig. 14), d'une humanité entière, dont l'essence repose sur le mouvement et la rythmique moderne des machines. 
Figure 14 : Agrandissement de la figure 4

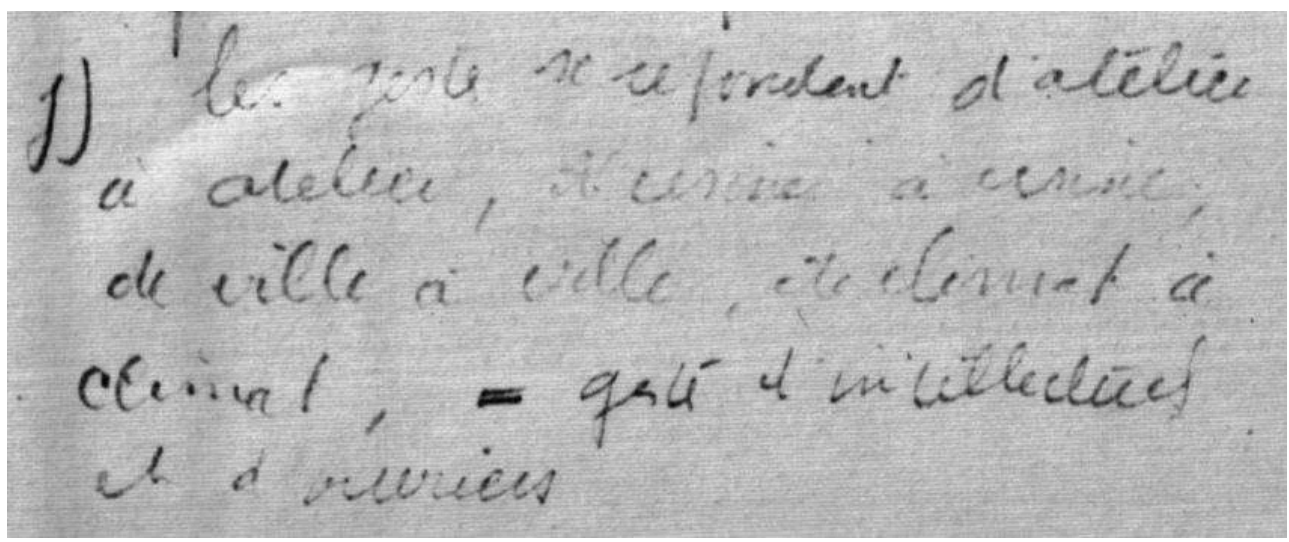

Cet agrandissement de la figure 4 (qui montre les ébauches de scénarios technographiques) permet de saisir comment Dekeukeleire, par le montage parallèle présent dans ses films technographiques, cherche à faire correspondre diverses dimensions de la société (usines, ateliers, villes, ouvriers et intellectuels) dans un discours symbolique sur le geste moderne.

La dimension explicative des films de commande se voit contrebalancée par leur structure poétique, signe d'une volonté de dépassement des cadres institutionnels. Dans une appréhension très conceptuelle de son propre art, c'est à travers le montage sensible que Dekeukeleire cherche à « atteindre l'esprit du machinisme » (ibidem : 80).

\section{La pensée machinique : construire le rêve moderne}

Sur le plan théorique, la technographie entretient des liens étroits avec une pensée spirituelle de la machine :

«Ce n'est pas le regard du technographe qui enregistre les aspects de notre monde, c'est un regard mécanique, [...] qui permet au technographe de capter tous les aspects de la vie, même ceux pour lesquels il ne ressent pas d'attrait. C'est également une mémoire mécanique qui retient les documents. Une mémoire parfaite qui garde aux images leur authenticité et leur fraîcheur et ne s'altère pas, fût-ce à des années de distance. L'appareil apporte aussi une imagination mécanique. Par le ralenti et l'accéléré, il analyse et synthétise nos regards comme ne pourrait le faire notre imagination. Mémoire et imagination mécaniques sont à la disposition de l'auteur pour monter, composer, pour rythmer l'enchaînement des images et des sons » (Dekeukeleire 1942 : 111).

41 Ces affirmations soulignent l'ambivalence du terme au regard d'une pratique du film industriel. Elles reflètent dans une moindre mesure la méthode appliquée à ce dernier par Dekeukeleire puisque l'imagination qui guide le montage final, bien qu'évoluant au gré du tournage, résulte avant tout d'un canevas de départ qui prédéfinit les prises de vue. Ici, Dekeukeleire oriente plutôt sa notion vers les termes de « ciné-œil » et « cinéesprit » abordés plus haut. Le technographe soumet son regard à celui de la caméra qui, dotée de son propre imaginaire technique, est présentée comme autonome. Cette idée dessine les contours de la "pensée machinique " évoquée en introduction. La notion est élaborée par Dekeukeleire pour désigner dans un double mouvement un archétype de pensée propre à l'ère industrielle et le rôle de la machine cinématographique, douée d'écriture et d'analyse, dans la construction des bases philosophiques de cet archétype.

Cet aspect de sa réflexion est en de nombreux points similaire avec les écrits de Jean Epstein, réalisateur français que le cinéaste belge côtoie et qui développe dès les années 
1920 une production écrite d'ampleur. Sa notion de «photogénie ${ }^{13}$ exercera notamment une influence considérable dans le domaine de la théorie du cinéma. Elle désigne la capacité de ce dernier à révéler les aspects poétiques du monde à travers sa technique : l'objectivité de la machine d'une part, le rythme du montage de l'autre. Epstein voit dans le film le moyen de forger une perception nouvelle du monde :

«Le rapport au temps se trouve reconfiguré par l'élaboration de représentations propres attestant la "pensée mécanique ", grâce aux procédés du ralenti, de l'accéléré, de l'inversion du film ou du gros plan. [...] Ce nouveau rapport donne au cinéma la capacité de renverser les antagonismes et de pulvériser les frontières, dans la philosophie et les sciences, entre des catégories fondamentales, telles continu et discontinu, esprit et matière, mouvement et immobilité, cause et fin. [...] Cette machine qui étire et condense la durée, qui démontre la nature variable du temps, qui prêche la relativité de toutes les mesures, semble pourvue d'une sorte de psychisme » (Thiéry $2014: 13$ ).

Les théories d'Epstein font de la technique cinématographique une entité à part entière, possédant - comme chez Dekeukeleire - une imagination propre : le cinéma devient une machine qui pense ${ }^{14}$ autrement. C'est le déplacement des fondements logiques de la réalité-même que le médium cinématographique provoque. Portée dans le champ psychique, cette pensée se réfère chez les deux réalisateurs aux structures cognitives de l'esprit. Si le premier apparente « le cinématographe [...] [à] une annexe de l'organe où généralement on situe la faculté qui coordonne les perceptions, c'est-àdire [...] [au] cerveau » (Epstein 2014:69), le second affirme dans son trente-quatrième carnet :

«Le [...] cinéma [...] nous restitue notre regard en dehors des contingences de temps et de lieu. Il nous restitue nos regards dans un ordre, un montage purement intérieur qui participe de nos rêves, qui participe du mécanisme de notre cerveau » (Dekeukeleire 1936: 74-75).

Chez les deux théoriciens, le cinéma écrit comme le cerveau fonctionne: l'œil machinique se prolonge dans l'œil humain et vice versa. Le cinéma éclate les relations à l'espace et au temps instaurés par le rationalisme, se présentant comme un retour à une logique perceptive, proche de la mémoire ou du rêve.

Cette vision du cinéma, éminemment poétique, semble s'éloigner des films institutionnels. Si elle se rapproche des documentaires sociaux cités plus haut, c'est dans le cinéma expérimental de Dekeukeleire qu'elle s'exprime le plus clairement.

Ce média ne peut être affiché ici. Veuillez vous reporter à l'édition en ligne http:// journals.openedition.org/itti/496

Dans un film comme Histoire de détective (1929), la caméra remplace l'œil humain (fig. 15), devenant protagoniste principale du film: c'est par elle qu'un détective, engagé pour suivre un époux en fuite, découvrira la vérité. Le film entier se structure autour de la vision de cette caméra, dans un montage éclaté de plans souvent a-humains qui s'approche du rêve. Cette déconstruction est poussée à son comble à travers le rythme d'Impatience (1928), œuvre la plus connue du cinéaste. Une trentaine de minutes plonge le spectateur dans un récit éclaté, où s'entremêlent le corps d'une femme et celui d'une moto en mouvement: la machine est le personnage principal du film. Le montage cadencé et les mouvements du véhicule dans le cadre y donnent à expérimenter un rythme et une manière de percevoir que l'on pourrait aisément qualifier de machiniques (fig. 16). 
La technographie ne peut que superficiellement être hiérarchisée. Tour à tour
critiques, conceptuelles ou institutionnelles, les différentes catégorisations de la notion
permises par l'analyse du discours de Dekeukeleire en font une notion flottante.
L'ambiguïté du terme crée un décalage entre pratique et idéologie qui souligne

La technographie ne peut que superficiellement être hiérarchisée. Tour à tour
critiques, conceptuelles ou institutionnelles, les différentes catégorisations de la notion
permises par l'analyse du discours de Dekeukeleire en font une notion flottante.
L'ambiguïté du terme crée un décalage entre pratique et idéologie qui souligne

La technographie ne peut que superficiellement être hiérarchisée. Tour à tour
critiques, conceptuelles ou institutionnelles, les différentes catégorisations de la notion
permises par l'analyse du discours de Dekeukeleire en font une notion flottante.
L'ambiguïté du terme crée un décalage entre pratique et idéologie qui souligne journals.openedition.org/itti/496

Si les premiers films de Dekeukeleire sont un terrain de recherche autour de la perception machinique, les films industriels qui leur succèdent ne leur sont pas contradictoires sur le plan théorique. Bien plutôt, ils en prolongent la réflexion dans un cadre social plus défini, à ceci près que le contexte institutionnel ne permet pas à Dekeukeleire de jouir d'une totale liberté esthétique. Dès ses débuts, le cinéaste vise à produire un montage qui puisse reproduire la structure cognitive de la mémoire afin d'atteindre l'inconscient de son spectateur par l'expérience sensible de l'image. Pour lui, le cinéma est un moyen de prendre connaissance du monde d'une manière presque directe, qui n'apparaît pas comme médiée: les spectateurs adhèrent instinctivement au rythme d'images qui semblent objectives par leur origine mécanique.

Des années 1930 aux années 1960, toutes ses recherches sur le cinéma ont convergé vers ce but et les films industriels se sont présentés à Dekeukeleire comme le lieu concret d'une mise en pratique de ses théories. Les épisodes poétiques récurrents qui structurent ces productions rappellent les débuts du cinéaste, à travers des séquences construites sur un montage parallèle de machines au travail (fig. 17).

Ce média ne peut être affiché ici. Veuillez vous reporter à l'édition en ligne http:// journals.openedition.org/itti/496

$\mathrm{Du}$ mouvement interne de l'image à celui du montage se dessine, dans ces enchaînements de plans, une poésie machinique à la cadence fragmentée - délinéarisée par les gros plans que l'on peut y rencontrer. Les raccords, qui reposent essentiellement sur le rythme du mouvement des machines dans les usines, renvoient à la symbolique du geste industriel abordé plus haut.

Le montage fait du cinéma un outil de propagande, idéal pour forger l'esprit de la civilisation des machines telle que Dekeukeleire le perçoit. La logique de résonnance rythmique et machinique entre les plans vise à s'approcher du rythme du cerveau pour mieux y intégrer une idéologie sociale, laquelle repose sur l'épanouissement du peuple dans son travail à l'usine. Désormais conscient de sa place au sein du travail et de la société, l'ouvrier par le cinéma comprend son rôle dans la collectivité. Le rêve individuel, façonné par les images sensibles, devient rêve collectif en ce qu'il est destiné aux masses. À travers la perception mécanique onirique de l'œil cinématographique, il revient au technographe de guider le peuple vers de nouvelles croyances collectives, d'écrire les fondements philosophiques de l'ère des machines, de bâtir les instincts d'une civilisation moderne.

\section{Conclusion}

Ce média ne peut être affiché ici. Veuillez vous reporter à l'édition en ligne http://

Images du travail, travail des images, 9 | 2020 
l'importance de mobiliser tous les pans de son discours: les écrits, mais aussi les méthodes employées et l'esthétique des différents films auxquels le terme paraît se rattacher. Les incohérences soulevées par ces analyses permettent de mesurer l'ambition théorique d'une telle notion. À défaut de cadres précis - qui au départ auraient dû être ceux du travail -, elle prend la forme d'une vision plus globale et tout à fait utopique du cinéma.

La notion répond aux postulats de Dekeukeleire sur l'art: née d'une économie industrielle, la technique cinématographique fait naître une forme d'art, dont le cinéaste attend qu'elle éduque la civilisation des machines. La notion doit être envisagée par le biais du documentaire social, s'appropriant l'idée de « sur-œil » dans le but de révéler une réalité humaine. Dans le champ du film industriel, elle glisse néanmoins en dehors de ces cadres en effaçant la figure humaine au profit d'une valorisation de l'usine et des appareils au travail. Par-là, Dekeukeleire rejoint l'esthétique machinique propre à ses premiers films. Dans une même veine, qui tient presque d'une obsession de la machine, les séquences poétiques des films institutionnels relèvent d'une tentative de faire intégrer au spectateur un esprit purement machinique, dans sa structure cognitive-même. Le montage rythme et construit alors, selon lui, les nouvelles croyances du peuple.

Une histoire des liens entre le cinéaste et ses commanditaires reste à faire pour comprendre jusqu'où transparaît la volonté artistique de Dekeukeleire. Si d'année en année, les épisodes poétiques se réduisent, c'est certainement parce que le cinéaste bénéficiait de moins en moins de liberté esthétique. Les théories, en revanche, se renforcent dans le sens d'un projet social assigné au film pour révéler le geste industriel de toute une époque. À aucun moment le réalisateur ne remet en question la forme ni la provenance de ses œuvres, qui restent pour lui la base de son projet.

Il est finalement affaire de propagande : Dekeukeleire entend s'introduire dans l'esprit des foules en les plongeant dans un rêve cinématographique, celui d'un épanouissement dans le travail à l'usine. Dans cette optique, il n'est pas étonnant que l'humain soit réifié et disparaisse au profit du système: il s'affirme non pas en tant qu'individu mais en tant que collectif. L'ouvrier participe à faire fonctionner le mécanisme plus général de l'usine, il alimente le rêve de toute la société industrielle. Sur base des théories propres au documentaire social, la réalité humaine qu'entend dévoiler la technographie est en fin de compte une réalité qu'elle construit de toutes parts. Elle traduit un rêve utopique - ou une obsession admirative pour les machines plus qu'une connaissance interne des hiérarchies sociales de l'usine. Elle montre certes la place de l'ouvrier, mais ne la questionne jamais, lui imposant le discours du système plus que ne laissant place au contingent (allant alors dans le sens contraire de la méthodologie énoncée dans les écrits).

58 En minimisant la dimension humaine, le projet de Dekeukeleire de recréer une cohésion sociale et une idéologie commune par le film industriel peut être considéré comme un échec dans sa pratique. Les films technographiques n'auront pas touché le public escompté, pas plus qu'ils ne seront parvenus à atteindre leurs buts idéologiques - répondant encore et toujours à des impératifs promotionnels d'institutions et entreprises plus spécifiques que le grand projet social auquel aspire le cinéaste. La recherche du geste et du rêve industriels restent chez lui éminemment conceptuelles, à l'image de la notion de technographie. 


\section{BIBLIOGRAPHIE}

Aubenas J. (1999), Dic doc. Le dictionnaire du documentaire. 191 réalisateurs, Bruxelles, Communauté française de Belgique.

Aumont J. (2002), Les Théories des cinéastes, Paris, Nathan.

Dekeukeleire C. (1932), « Le Cinéma contre lui-même », La Nouvelle Équipe 2-3, p. 219-227.

Dekeukeleire C., Willem Rombauts et Paul Werrie (1932), Réforme du cinéma, Bruxelles, René Henriquez.

Dekeukeleire C. (1942), L'Émotion Sociale. Réflexions sur un problème actuel, Bruxelles, La Maison du Poète.

Dekeukeleire C. (1947), Le Cinéma et la pensée. Le cinéma : art-clef de l'analyse du monde moderne. Son rôle dans la poursuite de l'aventure humaine, Bruxelles, Lumière (« Savoir »).

Epstein J. (2014), L'intelligence d'une machine, Le Cinéma du Diable et autres écrits [1945-1951], Paris, Independencia Éditions.

Lesuisse A.-F. (1998), « Charles Dekeukeleire ou l'utopie obligatoire » dans R. Odin (ed), L'Âge d'or du documentaire. Europe : Années cinquante. Tome 2 : Grande-Bretagne, Belgique, Pays-Bas, Danemark, Norvège, Suède, Paris, L'Harmattan, p. 55-68.

Remillet G. (2009), Ethno-cinématographie du travail ouvrier. Essai d'anthropologie visuelle en milieu ouvrier, Paris, L'Harmattan.

Sojcher F. (1999), La Kermesse héroïque du cinéma belge, 1896-1996. Des documentaires et des farces, 1896-1965 [tome 1], Paris, L'Harmattan.

Thiéry N. (2014), « L'incandescence d'une utopie théorique et artistique », dans J. Epstein, L'intelligence d'une machine, Le Cinéma du Diable et autres écrits, Paris, Independencia Éditions,11-25. Vertov D. (1984), Kino-Eye : the Writings of Dziga Vertov. Berkeley, University of California Press.

Vigo J. (2001), « Vers un cinéma social » [1930], dans D. Banda \& J. Moure, Le Cinéma : l'art d'une civilisation (1920-1960), Paris, Flammarion (« Champs Arts »), p. 224-227.

\section{Films cités}

La majorité des films de Dekeukeleire étudiés dans cet article peut être visionnée en ligne via la plateforme European Film Gateway : http://www.europeanfilmgateway.eu/fr.

Dekeukeleire C. (1928), Impatience. BE, N\&B, muet.

Dekeukeleire C. (1930), Witte Vlam. BE, N\&B, muet.

Dekeukeleire, C. (1930), Dixmude. BE, N\&B, muet.

Dekeukeleire, C. (1932), Visions de Lourdes. BE, N\&B, muet.

Dekeukeleire C. (1936), La Laine. BE, N\&B.

Dekeukeleire C. (1938), Images du travail. BE, N\&B.

Dekeukeleire C. (1938), Chanson de toile, BE, N\&B. 
Dekeukeleire C. (1949), 900 hommes (Usine Ford à Anvers). N\&B, muet.

Dekeukeleire C. (1950), Métiers d'Art de Flandre et de Wallonie. BE, N\&B.

Dekeukeleire C. (1954), Vers un monde nouveau. BE, N\&B.

\section{Archives}

Tous les carnets de Dekeukeleire sont disponibles à la consultation sur place, sur demande auprès de la bibliothèque de la Cinémathèque royale de Belgique. Pour cet article ont été utilisés les carnets suivants :

Carnet n³4, 30 Janvier 1936 au 23 mai 1936.

Carnet n62, 4 août 1946 au 15 février 1947.

Carnet $n^{\circ} 67,1^{\text {er }}$ novembre 1948 au soit 2, soit 8, soit 20 février 1949.

Carnet n69, du 5 juin 1949 au 21 novembre 1949.

\section{NOTES}

1. Ces films se nomment Combat de Boxe (1927), Impatience (1928), Histoire de détective (1929) et Witte Vlam (1930). Sur le plan historique, Dekeukeleire s'avère essentiellement associé aux trois premières œuvres qui se trouvent régulièrement diffusées dans le cadre de séances sur l'avantgarde au cinéma.

2. Respectivement nommées Le Mauvais đFil (1937) et Un petit nuage (1954).

3. La portée du réalisateur sur la cinématographie belge se situe plutôt du côté de ses films d'avant-garde et de ses tout premiers documentaires, encore marqués d'une forte expérimentation esthétique et structurelle. Les films promotionnels, s'ils peuvent comporter des parties poétiques proches de son travail d'avant-garde, ont été diffusés à petite échelle, au sein des institutions et industries concernées - ils sont donc aujourd'hui très peu connus.

4. Le cinéaste crée cette filmographie à thèmes en 1955. À défaut d'une liste exhaustive, certains exemples peuvent être cités : Thèmes d'inspiration (1938) est un film sur l'art, Le Fondateur (1947) traite d'histoire, Le Canal Albert (1938) et L'homme dans la ville (1951) explorent l'architecture. Terres Brûlées (1934) est un film à vocation ethnographique à propos de certaines régions d'Afrique que Dekeukeleire classe dans le domaine du folklore aux côtés, par exemple, de Processions et carnavals (1932), qui parcourt différentes manifestations traditionnelles belges. Enfin, Le Cuir (1937) Chanson de toile (1938), L'Usine aux champs (1945), À propos de fleurs (1952) ou encore Vers un monde nouveau (1955) documentent le travail industriel.

5. Les carnets de notes personnels de Charles Dekeukeleire, remplis entre 1931 et 1962, sont au nombre de cent-vingt-huit et représentent environ dix-mille feuillets d'archives.

6. Publiés à quelques années d'intervalle (les éditions belges ayant été gelées durant l'occupation allemande dans les années 1940), ces ouvrages sont pensés comme deux tomes d'une même réflexion sur le rôle social du cinéma : L'Émotion Sociale (1942) puis Le Cinéma et la pensée (1947) leurs références se trouvent dans la bibliographie de l'article.

7. Henri Storck et Charles Dekeukeleire se connaissaient, le premier s'étant formé au cinéma aux côtés du second. Bien que Storck ait bénéficié d'un rayonnement historique plus élevé que Dekeukeleire, les archives démontrent un intérêt relativement similaire pour leurs films des débuts. On place régulièrement les deux cinéastes au rang de pères fondateurs du cinéma belge. 
8. Dans leurs ouvrages sur le cinéma belge d'un côté et le documentaire de l'autre (cf. la bibliographie de cet article), Frédéric Sojcher et Jacqueline Aubenas soulignent les rapports entre cinéastes et commanditaires, qui pouvaient laisser une certaine liberté d'expression, bien que celle-ci doivent répondre à des exigences institutionnelles bien précises. Quelques films de commande de Dekeukeleire, à la forme parfois très originale, ont gagné des prix, tels que Thèmes d'inspiration (1938), et Noblesse du Bois (1954) et Germes de Lumière (1958).

9. C'est à travers un texte nommé "Réforme du cinéma », qui prend la forme du manifeste et qu'il présente aux côtés du poète Willem Rombauts et du journaliste Paul Werrie en 1932, que Dekeukeleire présente pour la première fois publiquement les enjeux d'une potentielle industrie cinématographique belge. Ils seront ensuite développés au long de sa carrière au gré de plusieurs conférences dont la trace a disparu mais dont les brouillons et la préparation subsistent dans les carnets.

10. Impatience (1928), notamment, se présente comme un film expérimental résolument hors de son propre temps et des avant-gardes qui lui sont contemporaines. Son esthétique remarquable fait de Dekeukeleire un cinéaste historiquement fascinant pour beaucoup.

11. Witte Vlam peut être vu à cette adresse: http://www.europeanfilmgateway.eu/detail/ Witte\%20vlam/crb::10f2ec2c5ffe8708bbb16e63a4344b74.

12. À visionner ici: http://www.europeanfilmgateway.eu/detail/Visions\%20de\%20Lourdes/ crb::a5cf8af3ae7aee6603cd533147920d4a.

13. Il faut ici renvoyer à La Photogénie de l'impondérable, texte majeur qu'Epstein publie en 1935.

14. Chez Epstein, comme chez Dekeukeleire, les termes de «pensée mécanique» ou " machinique » placent le cinéma sur le même plan que la pensée elle-même. Les titres de leurs ouvrages sur le cinéma, dont les théories se rapprochent fortement et qui sont publiés à quelques mois d'intervalle, sont à ce sujet très éclairants : L'Intelligence d'une machine (Epstein, 1946) et Le Cinéma et la pensée (Dekeukeleire, 1947).

\section{RÉSUMÉS}

Cet article vise à définir et interroger la notion de «technographie» développée par le réalisateur belge Charles Dekeukeleire (1905-1971) pour désigner les films industriels que celui-ci a produits à partir des années 1930-1940. Une analyse des discours théoriques puis des formes filmiques du cinéaste permet de comprendre que le terme dépasse son champ d'origine pour s'échapper dans le domaine, plus conceptuel, de la théorie du cinéma. La technographie se situe à la croisée d'idéologies et de méthodologies diverses entre films sur le travail à l'usine, films anthropologiques et films expérimentaux - assignant un rôle social à l'écriture technique du cinéma.

This article aims at introducing, defining and questioning the notion of "technography" developed through Charles Dekeukeleire's writings. It refers to the Belgian filmmaker's industrial works, produced from the 1930s and 1940s onwards. The analysis of his theoretical texts and filmic forms allows one to understand that "technography" goes beyond its original meaning so as to integrate the more conceptual field of film theory. Technography is at the junction of different ideologies and methodologies between industrial, anthropological and experimental films - defining cinema as a sensible technique endowed with a social role. 
INDEX

Mots-clés : technographie, film industriel, cinéma belge, théorie du cinéma, machinisme

Keywords : technography, industrial film, belgian cinema, film theory, industrial civilisation

\section{AUTEUR}

\section{MATHILDE LEJEUNE}

Mathilde Lejeune est doctorante en quatrième année de thèse en Études Cinématographiques aux Universités de Lille et de Lausanne sous la direction conjointe d'Edouard Arnoldy et de Laurent Le Forestier. Bénéficiant d'un contrat doctoral attribué par l'Université de Lille (2016-2019), elle se concentre sur le fonds d'archives de Charles Dekeukeleire, cinéaste belge (1905-1971) déposé à la Cinémathèque royale de Belgique, à partir duquel elle entend faire l'archéologie des théories sur le cinéma du réalisateur. Ses intérêts académiques portent sur le cinéma d'avant-garde, la théorie du cinéma des années 1930-1940, les rapports entre cinéma et mémoire et les questions d'écriture cinématographique. 